\title{
Prevailing approaches in chronic liver disease hemostasis
}

\begin{abstract}
Based on the emerging knowledge of the complexity and the flexibility of the systems involved in maintaing hemostasis in health and in disease, the tenet of coagulation in cirrhosis has undergone significant revision in the last 18 years. Recent attention has focused on global assays to better guide therapeutic decisions tailored to each patient's clinical and hemostatic status. This new concept generates consequences in therapeutic interventions whether as prophylaxis or as salvage for acute events. There is yet no optimal management strategies for the critical clinical outcomes (bleeding or thrombosis) in cirrhotics neither as prophylaxis nor as therapy.
\end{abstract}

Keywords: cirrhosis, hemostasis, global assays, transfusion medicine
Volume 8 Issue 5 - 2020

\author{
Dragica Vucelic,' Nikica Grubor',2 \\ 'Clinic for Digestive Surgery, Clinical Center of Serbia, Serbia \\ ${ }^{2}$ Faculty of Medicine, University of Belgrade, Serbia
}

Correspondence: Dragica Vucelic, MD, PhD, Transfusion Medicine Department, Clinic for Digestive Surgery, Clinical Center of Serbia, Belgrade, Koste Todorovic 6, I 1000 Belgrade, Serbia,Tel+38I II 3663757, Email vucelicd@gmail.com

Received: September 02, 2020 | Published: September 30 2020
Abbreviations: ADAMTS13, a specific vWF-cleaving metalloprotease; AT, antithrombin; AUC, area under the curve; CLD, chronic liver disease; CPs, Child-Pugh score; ETP, endogenous thrombin potential; F, factor; FFP, fresh frozen plasma; INR, international normalized ratio; LMWH, low molecular weight heparin; MCF, maximum clot firmness; PAI-1, plasminogen activator inhibitor-1; PC, protein C; PCC, prothrombin complex concentrate; PH, portal hypertension; PS, protein S; PT, prothrombin time; PVT, portal vein thrombosis; rFVIIa: recombinant activated factor VII; ROTEM, rotational thromboelastometry; TAFI, thrombin activatable fibrinolysis inhibitor; TEG, thromboelastography; TGA, thrombin generation assay; TIPS, trans-jugular intrahepatic portosystemic shunt; tPA, tissue plasminogen activator; UGIB, upper gastrointestinal bleeding; VAK, vitamin K antagonist; VTE, viscoelastic testing; vWF, von Willebrand factor

\section{Background}

'Science is truthful in a very special, permanently temporary way. One holds on to the powerless erroneous facts that used to be true when learned'. Karl Popper.

The net result of impaired hemostasis in in patients with chronic liver disease (CLD) involve multiple defects in its three main aspects: primary hemostasis, coagulation and fibrinolysis. According the current prevailing concept, patients with cirrhosis are ultimately effectively 'rebalanced' with regard to both procoagulant and anticoagulant pathways (both drivers are lowered), and most of these patients remain in a complex, dynamic, and fragile balanced state of hemostasis that is provided by acting of compensatory mechanisms. ${ }^{1-4}$ The defects in platelet number and function are often compensated by supraphysiological von Willebrand factor (vWF) levels, and (at least partly) by reduced levels of ADAMTS13 (a specific vWF-cleaving metalloprotease). The acquired deficiencies in the procoagulant pathways are counterbalanced by deficiencies of such naturally occuring anticoagulants as antithrombin (AT), proteins $\mathrm{C}(\mathrm{PC})$ and $\mathrm{S}$ (PS), coupled with the marked increase in plasma factor VIII (FVIII). The fibrinolytic system may also be in a precarious balance due to concomitant affection in both profibrinolytic [high tissue plasminogen activator (tPA), low antiplasmin and thrombin activatable fibrinolysis inhibitor (TAFI) and anti-fibrinolytic (low levels of plasminogen and high levels of plasminogen activator inhibitor (PAI-1)] directions. ${ }^{1,5-7}$ However, in patients with and-stage liver disease the hemostatic balance is unstable because of decreased reserve, and small perturbations can lead to bleeding or thrombosis depending on the prevailing circumstantial risk factors, which may change with the clinical status of the patients. ${ }^{1,5,7,8}$

At a present routine hemostasis tests cannot be advocated for prediction of bleeding risk related to invasive/surgical procedures or the occurrence of gastrointestinal bleeding. ${ }^{4,5}$ As a practical metter, in cirrhosis patients there is a growing interest in using more global hemostatic assays that are available and rapidly enough to practicing physician for clinical outcomes prediction. ${ }^{9,10}$

\section{Hemostasis examination in liver disease}

The shortcomings of standard hemostasis tests for bleeding risk assessment in patients with CLD have been extensively reviewed elsewhere. ${ }^{1-3,5,8,11}$ The failure of available standard tests to reflect complex hemostatic balance and predict clinical outcome in this particular patient population has led to a significant interest in global tests of hemostasis such as thrombin generation assay (TGA) and viscoelastic testing (VET). ${ }^{12-14}$ TGAs and VETs represent manifold methods which determine different physical properties in diverse circumstances (centrifuged plasma vs. whole blood). Instead of looking at the whole thrombin generation dynamics, VETs are dependent on thrombin generation measuring its effect on clot formation. ${ }^{12}$ Unlike relatively time-consuming TGAs, VETs are a point-of-care methods which allow real-time reports. VETs have clot formation as the endpont [maximum clot firmness (MCF) is taken to represent the ultimate strength of the clot] which offers advantage to TGAs or standard tests such as prothrombin time (PT) which have thrombin generation or the time point of fibrinogen to fibrin conversion in plasma as the endpoint. ${ }^{6,12-14}$ VETs have unique ability to study the clotting process starting with fibrin formation and continue through the clot retraction and fibrinolysis reflecting overall clot elasticity. ${ }^{12,14}$ A potential of VETs as appropriate tool for detection of 
FXIII deficiency in cirrhotics has been recently suggested. ${ }^{15}$ However, VETs lack activation of the PC system, and are insensitive for primary hemostasis. ${ }^{6}$ The two most widely used commercially availible VETs are thromboelastography (TEG) and rotational thromboelastometry (ROTEM) sharing similar measuring principle. ${ }^{9}$

Numerous studies ${ }^{16-20}$ reported the TGAs indicated preserved or even increased coagulation profile in plasma from cirrhotics when TGAs were performed in the presence or withouth thrombomodulin, suggesting a potential for hypercoagulability. There is still scarce information on usefulness of global hemostatic assays in homogenous group of patients with cirrhosis related to different etiologies.

Belgrade team ${ }^{10}$ performed three approaches of hemostasis, including standard hemostasis tests, ROTEM and TGA (unmodified endogenous thrombin potential - ETP assay in the absence of thrombomodulin; a commercially available assay Innovance $($ ) in samples from patients with alcoholic cirrhosis $(n=60)$ and normal subjects $(n=50)$. A large proportion of patients was found to be hypocoagulable as suggested by the standard tests and ETP. In ETP assay, hypocoagulable thrombin generation profile was observed in $91 \%$ and $81.6 \%$ of patients, respectively. Possible explanation for this observation is absence of soluble thrombomodulin in ETP measurement, as it was formerly suggested by Tripodi et al. ${ }^{16}$ Using unmodified TGA (without thrombomodulin) in heterogenous group of patients with different causes of cirrhosis, Gatt et al. ${ }^{17}$ found only $8.2 \%$ of patients had reduced ETP levels. In modified TGA (with adding Protac: a non-physiologic activator of PC), Gatt et al. ${ }^{17}$ also found the coagulation system in plasma of patients with cirrhosis is procoagulant However what the authors did not adress in their study is whether this assumption is steel holds true when different cirrhosis etiologies are under examination as homogenous groups rather than groups of mixed etiologies. Kleinegris et al. ${ }^{19}$ also explored patients with all-cause cirrhosis, and found a prothrombotic state in their plasma even in the TGA measurements conducted without thrombomodulin which is not in line with Tripodi et al ${ }^{16}$ observation. These contrary results were explained by different concentration of added phospholipids $(4 \mu \mathrm{M}$ vs. $0.5 \mu \mathrm{M})$ to the thrombin generation measurements. Furthermore, Kleinegris et al. $^{19}$ reported hypocoagulable profile in ROTEM associated with increased severity of cirrhosis. In contrast, in Belgrade team study ${ }^{10}$ more than a half of the patients had normal clot firmness assessed by ROTEM. In this study ${ }^{10}$ there was a greater proportion of patients classified with Child-Pugh score $(\mathrm{CPs}) \mathrm{B}(\mathrm{n}=28)$ and $\mathrm{CPsC}$ $(\mathrm{n}=27)$, respectively in comparison with Kleinegriset et al. ${ }^{19}$ mixed cohort study $[\mathrm{n}=73 ; \operatorname{CPsB}(\mathrm{n}=15)$ and $\operatorname{CPSC}(\mathrm{n}=6)$, respectively)] in which the majority of patients had well compensated disease [CPsA $(n=52)]$. Lentschener et al. ${ }^{21}$ detected hypocoagulability on traditional testing and ROTEM (correlated to liver dyfunction) while TGA in the presence of thrombomodulin demonstrated preserved or even increased thrombin generation (inversely correlated to the degree of liver dysfunction). Authors concluded that ROTEM may not be appropriate for assessing the coagulation status in cirrhotics while may present a reliable index of the degree of liver failure. In contrast, Vucelic et $\mathrm{al}^{10}$ found a majority of coagulation proteases better correlated with ETP compared to MCF. As expected, the prothrombin was the most consistent determinant of the amount of thrombin generated, as quantified by area under the curve - AUC ( $\mathrm{r}=0.822$; $\mathrm{p}<0.001)$. This result is coherent with observation that any variation in prothrombin level is reflected in the pattern of thrombin formation. ${ }^{22,23}$ The major predictors for MCF were platelets count and fibrinogen $(\mathrm{r}=0.693 ; \mathrm{p}<0.001$ and $\mathrm{r}=0.594 ; \mathrm{p}<0.001$, respectively). These results indicate that platelets and fibrinogen rather than levels of coagulation factors were crucial for clot formation. In contrast to additional study ${ }^{24}$ comparing traditional coagulation testing and ROTEM in cirrhotics and healthy controls, in this study ${ }^{10}$ significant correlations of MCF with disease severity estimated by CPs were not found. Moreover, the correlations between MCF and biochemical liver function was not found for albumin and total bilirubin, and only a weak correlation between cholinesterase and EXTEM-MCF was observed. On the other hand, ETP parameters correlated with all explored biochemical variables. Among investigated parameters of biochemical liver function, the best correlation was obtained for cholinesterase $(\mathrm{r}=0.610$; $\mathrm{p}<0.001)$. On the whole, ETP parameters correlated with both CPs and synthesis function of the liver. Thus, authors have hypothesized that ETP could be potentially useful indicator of disease severity/liver synthesis function in cirrhotics since AUC represents what happens after PT. Unlike ETP, results suggested the clinical utility of ROTEM as a functional assay in providing supplemental information such as MCF and clot stability over time, which cannot be derived from ETP. In a view of the conflicting results provided in separate studies further exploration is warranted. Since TGA is time consuming and reagents are not yet standardized, efforts at standardization and development of a point-of-care whole blood assay are in progress. ${ }^{25}$

More recent studies ${ }^{19,21,24,26-28}$ have explored the usefulness of VETs in investigating hemostasis in CLD (some results discussed previously). Of note, Vucelic et al. ${ }^{26}$ assessed thromboelastometric FIBTEM-MCF test (designed to measure fibrin-based clot firmness in the presence of a platelet inhibitor) in regard to its relevance in detection of qualitative fibrinogen disorders in comparison with results obtained by the Clauss fibrinogen method in 60 patients with alcoholic and 24 patients with cholestatic cirrhosis [(CPs) A, n=24; B, $\mathrm{n}=32 ; \mathrm{C}, \mathrm{n}=28]$ compared to 50 healthy individuals. In all CPs groups, fibrinogen levels strongly correlated with FIBTEM-MCF and tended to decrease as liver disease advanced. However, in the subgroup of 16 patients with reduced FIBTEM-MCF $(<9 \mathrm{~mm})$, seven had normal fibrinogen levels (range 1.9-3.98 g/L) which may be affirmative as regards the presence of qualitatively altered fibrinogen in these cases. In a study exploring 51 patients with cirrhosis, Tripodi et al. ${ }^{24}$ demonstrated that FIBTEM-MCF was not useful in distinguishing cirrhosis patients from healthy individuals. The authors described irregular FIBTEM-MCF in 8/51 (16\%) patients, similarly to Vucelic et al. ${ }^{26}$ findings of decreased FIBTEM-MCF in 16/84 (19\%) patients. However, Tripodi et al. ${ }^{24}$ did not focus on comparison of standard fibrinogen measurement with clot firmness in a subgroup of patients with abnormal FIBTEM-MCF. Taking together, FIBTEM test could be considered as a promising tool for fibrinogen assessment in patients with cirrhosis. The advantage of the FIBTEM method is whole blood, real-time measurement which can provide valuable information in situations with high clinical priority such as bleeding complications, the need for preoperative assessment of fibrinogen status as well as monitoring during and after invasive/surgical procedures. However, the question remains with regard to the threshold value of FIBTEM$\mathrm{MCF}$ as a predictor of bleeding in cirrhotics. On the whole, there is still no evidence for cut-off VET parameters values (the most usefull parameter is MCF representing summation of primary and secondary hemostasis) to identify patients at risk for hemorrhagic events in both cirrhotics udergoing invasive procedures or bleeding subjects as well as optimal MCF values for competent hemostasis.

Although debated as to significance, accelerated fibrinolysis has long been recognized clinical feature of cirrhosis resulting in an increased bleeding tendency (diffuse oozing from mucosal surfaces, persistant postoperative or delayed puncture site bleeding), especially 
when cirrhotics are under physiologic stress (e.g. during infection, surgery and bleeding or ascites fluid egress). ${ }^{3,7,8,25}$ More global assays have been examined as a tool to identify hyperfibrinolysis and to optimize rescue approaches when needed ${ }^{9}$. In liver transplant patients, better sensitivity of tissue factor-triggered ROTEM tests compared to contact-activated kaolin-TEG in identifying hyperfibrinolysis was demonstrated $(p<0.001)$. In addition, it was shown that inhibition of platelet-fibrin interaction in FIBTEM enhanced sensitivity to hyperfibrinolysis detection compared with EXTEM. ${ }^{29}$

\section{Management of bleeding complications}

Undoubtedly, end-stage liver disease is considered as being bleeding disorder which may have prominent bleeding symptoms, particularly in the gastrointestinal tract. ${ }^{7}$ In cirrhotics, spontaneous bleeding events relate to primary hemostasis defects are most frequently encountered while haemorrhage as a consequence of impaired coagulation is uncommon. ${ }^{6,30}$ Bleeding tendency observed in patients with advanced liver disease does not find its main cause in defects of haemostasis but rather among underlying conditions that favour hemorrhage such as haemodynamic alterations of portal hypertension $(\mathrm{PH})$, endothelial dysfunction, bacterial infection, and renal failure. Three lesions most commonly responsible for nonvariceal spontaneous upper gastrointestinal bleeding (UGIB) are gastric/duodenal ulcer and portal hypertensive gastropathy. ${ }^{30,31}$ The most common causes of lower gastrointestinal bleeding in cirrhotics are portal hypertensive colopathy, ileal and colorectal varices and hemorrhoids (all caused by PH). ${ }^{32}$ The clinically most relevant bleeding problem and the major cause of death in cirrhotics is acute bleeding from ruptured esophageal varices, accounting for $70 \%$ of UGIB cases. ${ }^{33}$ As a fact, there have been few data supporting the role of systemic hyperfibrinolysis, endogenous heparin-like substances and primary hemostasis in the occurance of variceal hemorrhage. ${ }^{8,34,35}$

No general guidelines are available for pharmacological interventions to control bleeding in patients with liver disease. Recommendations are now focused on individualizing care for management of hepatic coagulopathy. Approaching hemostatic issues in cirrhosis must be tailored to the clinical scenario and definition of particular coagulation abnormalities present at a given timepoint at a patient level. When bleeding does occur, different procoagulant therapies in modifying haemostasis should be considered such as vitamin $\mathrm{K}$, fresh frozen plasma (FFP), cryoprecipitate, fibrinogen concentrate, platelets, antifibrinolytic agents, desmopressin, prothrombin complex concentrate (PCC), recombinant activated factor VII (rFVIIa), and red blood cells..$^{25,30,36}$

There is a little consensus regarding coagulation management for acute variceal bleeding episode. Replacement with red cell transfusion should be performed with a caution to avoid deterioration of $\mathrm{PH}$ and increase in rebleeding rate. The goal is to maintain hemoglobin level at $70-90 \mathrm{~g} / \mathrm{L}$ in the context of ongoing hemorrhage. There are no specific directions regarding FFP, cryoprecipitate/fibrinogen concentrate and platelets transfusion for variceal bleeding patients, although platelet count exeeding $56 \times 10^{9} / \mathrm{L}$ is suggested. ${ }^{8,33,37}$ In active bleeding patients, fibrinogen levels below $120-150 \mathrm{mg} / \mathrm{dL}$ is proposed as a critical value for fibrinogen replacing. ${ }^{4,25}$ FFP should be avoided because fluid overload and iatrogenic increase in portal pressure may paradoxically increase rebleeding risk. ${ }^{6,8}$ The role of rFVIIa as a rescue agent in uncontrolled variceal hemorrhage remains to be contemplated. ${ }^{8,37}$ The current largely accepted outlook stands against general use of rFVIIa in active variceal bleeding episode because of substantially negative clinical trial data and its high expense. ${ }^{36}$ Interestingly, concerning rFVIIa failure to control bleeding there are no available data or comments with regard to hemostasis parameters other than decreased prothrombin activity, factor levels, reduced platelet count, and hematocrit. Therefore, fundamental reasons for rFVIIa failure to control hemorrhage in cirrhotics experiencing acute UGIB may be attributed to possible presence of fibrinogen disorders and/or hiperfybrinolysis (local and systemic). There are strong in vitro data demonstrating the presence of increased local level of fibrinolytic activity in upper gastrointestinal tract. ${ }^{38,39}$ Patients with cirrhosis were reported to have higher fibrinolytic activity of the mucosa of stomach and esophagus than those without cirrhosis. The presence of more increased fibrinolytic activity of the esophageal mucosa was determined in cirrhotics with varices in comparison to those without esophageal varices. ${ }^{40}$ It is biologically reasonable to await that the degree of local hyperfibrinolysis in cirrhotics may be related to worsening of bleeding in acute haemorrhagic episodes, and presumably with increased risk for variceal rebleeding. In this perspective, increased systemic fibrinolytic activity could be recognized as additional aggravating factor for bleeding onset. Along with specific therapies, more emphasis should perhaps be laid upon examination the efficacy of antifibrinolytic therapy in the treatment of acute variceal hemorrhage as well as in the context of secondary prevention of recurrent bleeding. Patients thus would be likely benefit from a drug that could amend both local and systemic hyperfibrinolysis given that these measures can improve clot stability (for reduction of local fibrinolytic activity a drug could be administer via a nasogastric tube by using a syringe for injection). We beleive that clinical studies in order to evaluate the usefulness of therapeutic fibrinogen and antifibrinolytics administration at the time of active variceal bleeding treated with rFVIIa seems to worth the effort. Of note, correction of coagulopathy is not addressed and no hemostatic agent have been specifically commented by established authorities in recent practice guidances that summarize the most important aspects for an optimal management of varices and variceal hemorrhage. ${ }^{33,37,41}$

\section{Prophylactic correction of abnormal hemostasis before invasive procedures or surgery}

It is widely held view that procedure-related bleeding is occasional. ${ }^{42}$ The primary intention should be focused at defining a coagulation profile at a patient level as it can vary greatly among cirrhotics. ${ }^{8,42}$ During the low or moderate risk procedures prophylaxis is not routinely recommended for stable cirrhotics with platelets $>50 \times 10^{9} / \mathrm{L}^{4,25}$ Although target levels for pre-procedural prophylaxis remain unclear, patients who undergo high-risk procedures should receive pre-procedural prophylaxis with platelet concentrates and fibrinogen at a levels less than $50 \times 10^{9} / \mathrm{L}$ or of $120-150 \mathrm{mg} /$ $\mathrm{dL}$, respectively, ${ }^{4,25,36}$ Oral thrombopoetin agonists (eltrombopag, avatrombopag, lusutrombopag) are potential alternatives for platelet transfusions to increase platelets prior invasive procedures with moderate or high risk although such treatment is not generally advisable at this time because of the relative risk of thrombotic events. . $^{45,42}$

Among non-transfusional pharmacological agents within clinician's armamentarium for preventing or treating bleeding episodes, the utility of desmopressin in cirrhotics is not definitively defined. At the time of writing this manuscript, it is commonly been assumed that in patients with cirrhosis the use of desmopressin does not appear to significantly alter risk of bleeding with the exclusion of those undergoing dental extraction. ${ }^{25}$ Altough routine administration 
of vitamin $\mathrm{K}$ is not recommended in cirrhotics ${ }^{42}$, intravenous vitamin $\mathrm{K}$ repletion would aid in hemostasis protein production. ${ }^{36}$ The role of PCC in prophylaxis in patients with CLD is currently undefined. ${ }^{36}$ The use of antifibrinolytic therapy in liver surgery and other clinical circumstances at high risk for excessive bleeding may be helpful in a prophylactic setting, particulary in patients who have decompensated cirrhosis with ascites. ${ }^{5,6,36,42}$ In patients undergoing dental extraction, mouth washes with tranexamic acid after procedure should be considered as saliva has fibrinolytic properties that might affect hemostasis in oral cavity. ${ }^{36,42}$

Treatment of active infection and acute kidney injury are very important considerations in preventing bleeding complications, given that both can significantly alter hemostasis..$^{4,5,25,42}$ An important strategy to prevent bleeding during invasive procedures or surgery is to carry out volume restriction and forced diuresis to maintain a low splanchic and portal pressure and a low total circulating volume while sufficiant tissue perfusion can be acomplished through the use of vasoconstritors. ${ }^{5}$

\section{Treatment of non-surgical blood loss during surgery or other invasive procedures}

Among the pharmacological agents, low-volume prohemostatic PCC could be considered to achieve successful clinical hemostasis in patients with cirrhosis who are actively bleeding. ${ }^{36}$ The use of rFVIIa as a 'rescue' agent in patients experiencing ongoing haemorrhage remains to be adequately studied..$^{25,36}$ In patients with active bleeding, replacement of low/altered fibrinogen with fibrinogen concentrate/ cryoprecipitate should be considered. ${ }^{4,25,36}$ Antifibrinolytics may be applied to treat bleeding in hepatic surgery or persistent postoperative wound/mucosal/puncture site bleeding that could be sign of hyperfibrinolysis. ${ }^{8,42}$ Guidance with VETs may be useful in the context of active bleeding in patients with CLD. ${ }^{4,5}$ General practitioners are advised to take every effort in the assessment for evidence and treatment of renal failure and infection if these complications are present in active bleeding patients. ${ }^{42}$

\section{Management of thrombotic complications}

Clinicians are left with several unanswered questions on thrombosis management in cirrhotics: (i) which risk factors identify patients in whom thromboprophylaxis is likely to be beneficial?; (ii) what is the best choice for anticoagulant agent with regard to safety and effectiveness in either prevention or treatment of thrombosis? (iii) what is the most reliable method for treatment monitoring?; (iiii) which are the optimal intensity and duration of thromboprophylaxis in non-transplant candidates (in candidates for liver transplantation, anticoagulation should be maintanied until transplantation)? $?^{6,42}$

To prevent venous thromboembolism (VTE), the most common practice being the use of low molecular weight heparins (LMWHs) or vitamin K antagonists (VAKs). Standard pharmacologic VTE prophylaxis is recommended for hospitalized cirrhotics, especially when exposed to major surgery and prolonged immobilization. The use of LMWH to prevent portal vein thrombosis (PVT) and decrease the risk of hepatic decompensation (anticoagulation could have impact on slowing down liver fibrosis) is advisable. Patients with established PVT should be treated by LMWH or VKA, although unpredictable pharmacokinetics of these drugs in cirrhotics should be under consideration. ${ }^{6,8,25,42}$ Since international normalized ratio (INR) is already prolonged in many patients with advanced disease, the appropriate target range is unclear. ${ }^{5,6} \mathrm{~A}$ significant disadvantage of LMWH administration lies in a fact that anti-Xa assays can be misleading in liver disease giving substential underestimation of the true LMWH circulating mass. ${ }^{5,6,25}$ From a theoretical point of view, as liver disease is commonly associated with both AT deficiency and raised FVIII levels the possibility of 'heparin resistance' need to be considered in each individual independently. ${ }^{43,44}$ More recently, transjugular intrahepatic portosystemic shunt (TIPS) is recommended to be safe and effective to revascularize the portal venous system in cirrhotics. ${ }^{25}$ Treatment for new diagnoses of VTE should essentially follow the same protocol as for PVT. ${ }^{8}$ For the present time, use of direct oral anticoagulans is supported exclusively for well-compensated patients, but their safety and effectiveness remain to be evaluated. ${ }^{25,42}$

\section{Conclusion}

Patients with liver disease pose a significant challenge to treating physicians. Efforts of modern hemostatic research should be focus on defining reliable hemostasis tests to predict clinical outcomes and thereby optimize hemostatic approaches with regard to either bleeding or thrombosis. Assessment of bleeding risk among patients with liver disease in the perioperative period or after other invasive procedures is one of the most important challanges in clinical practice. A significant amount of work remains to be done to improve anticoagulation therapies for the prevention and treatment of thrombosis in patients with liver disease.

\section{Authors contributions}

D. Vucelic and N. Grubor contributed equally to the development of the concept and design of this work. D Vucelic wrote the initial version of the manuscript and N. Grubor revised the paper. No funding for writing this review was obtained.

\section{Acknowledgments}

None.

\section{Conflicts of interest}

The authors declare they have no conflict of interest to report.

\section{References}

1. Lisman T, Porte RJ. Rebalanced hemostasis in patients with liver disease: evidence and clinical consequences. Blood. 2010;116(6):878-885.

2. Tripodi A, Primignani M, Mannucci PM. Abnormalities of hemostasis and bleeding in chronic liver disease: the paradigm is challenged. Intern Emerg Med. 2010;5(1):7-12.

3. Northup PG, Caldwell SH. New concept of coagulation and bleeding in liver disease. Intern Emerg Med. 2010;5:3-6.

4. Tripodi A, Primignani M, Mannucci PM, et al. Changing concepts of cirrhotic coagulopathy. Am J Gastroenterol. 2017;112(2):274-281.

5. Weeder PD, Porte RJ, Lisman T. Hemostasis in liver disease: implications of new concept for perioperative management. Transfus Med Rev. 2014;28(3):107-113.

6. Lisman T, Porte RJ. Pathogenesis, prevention, and management of bleeding and thrombosis in patients with liver disease. Res Pract Thromb Haemost. 2017;1(2):150-1161.

7. Tripodi A, Mannucci PM. The coagulopathy of chronic liver disease. $N$ Engl J Med. 2011;365(2):147-156.

8. Northup PG, Caldwell SH. Coagulation in liver disease: a guide for the clinician. Clin Gastroenterol Hepatol. 2013;11(9):1064 -1074. 
9. Davis JPE, Northup PG, Caldwell SH, et al. Viscoelastic testing in liver disease. Ann Hepatol. 2018;17(2):205-213.

10. Vucelic D, Jesic R, Zivotic M, et al. Diverse utility of endogenous thrombin potential and thromboelastometry in the assessment of patients with alcoholic cirrhosis. Hematol Transfus Int J. 2015;1(2):34-44.

11. Mannucci PM. Abnormal hemostasis tests and bleeding in chronic liver disease: are they related? No. J Thromb Haemost. 2006;4:721-723.

12. Nair SC, Dargaud Y, Chitlur M, et al. Tests of global haemostasis and their applications in bleeding disorders. Haemophilia. 2010;16(Suppl 5):85-92.

13. Van Veen JJ, Gatt A, Makris M. Thrombin generation testing in routine clinical practice: are we there yet?. Br J Haematol. 2008;142(6):889-903.

14. Othman M. Global hemostasis: new approaches to patient diagnosis and treatment monitoring. Semin Thromb Hemost. 2010;36(7):695-698.

15. Bedreli S, Sowa JP, Malek S, et al. Rotational thromboelastometry can detect factor XIII deficiency and bleeding diathesis in patients with cirrhosis. Liver Int. 2017;37(4):562-568.

16. Tripodi A, Salerno F, Chantarangkul V, et al. Evidence of normal thrombin generation in cirrhosis despite abnormal conventional coagulation tests. Hepatology. 2005;41(3):553-558.

17. Gatt A, Riddell A, Calvaruso V, et al. Enhanced thrombin generation in patients with cirrhosis -induced coagulopathy. J Thromb Haemost. 2010;8(9):1994 -2000.

18. Youngwomn N, Kim JE, Lim HS, et al. Coagulation proteins influencing global coagulation assays in cirrhosis: hypercoagulability in cirrhosis assessed by thrombomodulin-induced thrombin generation assay. Biomed Res Int. 2013;2013:856754

19. Kleinegris $\mathrm{MC}$, Bos $\mathrm{MH}$, Roest $\mathrm{M}$, et al. Cirrhosis patients have coagulopathy that is associated with decreased clot formation capacity. $J$ Thromb Haemost. 2014;12(10):1647-1657.

20. Groeneveld D, Porte RJ, Lisman T. Thrombomodulin-modified thrombin generation testing detects a hypercoagulable state in patients with cirrhosis regardless of the exact experimental conditions. Thromb Res. 2014;134(3):753-756.

21. Lentschener C, Flaujac C, Ibrahim F, et al. Assessment of haemostasis in patients with cirrhosis: Relevance of the ROTEM tests? Eur $J$ Anaesthesiol. 2016;33(2):126-133.

22. Monroe DM, Hoffman M. What does it take to make the perfect clot? Arterioscler Thromb Vasc Biol. 2006;26(1):41-48.

23. Allen GA, Wolberg AS, Oliver JA, et al. Impact of procoagulan concentration on rate, peak and total thrombin generation in a mode system. J Thromb Haemost. 2004;2:402-413.

24. Tripodi A, Primignani M, Chantarangkul V, et al. The coagulopathy of cirrhosis assessed by thromboelastometry and its correlation with conventional coagulation parameters. Thromb Res. 2009;124(1):132-136.

25. Intagliata NM, Argo CK, Stine JG, et al. Concepts and controversies in haemostasis and thrombosis associated with liver disease: proceedings of the 7th international coagulation in liver disease conference. Thromb Haemost. 2018;118(8):1491-1506.

26. Vucelic D, Jesic R, Jovicic S, et al. Comparison of standard fibrinogen measurement methods with fibrin clot firmness assessed by thromboelastometry in patients with cirrhosis. Thromb Res. 2015; $135: 1124-1130$
27. Shin KH, Kim IS, Lee HJ, et al. Thromboelastographic evaluation of coagulation in patients with liver disease. Ann Lab Med. 2017;37(3):204 212.

28. Lloyd -Donald P, Vasudevan A, Angus P, et al. Coagulation in acutely ill patients with severe chronic liver disease: insights from thromboelastography. J Crit Care. 2017;38:215-224.

29. Abuelkasem E, Lu S, Tanaka K, et al. Comparison between thrombelastography and thromboelastometry in hyperfibrinolysis detection during adult liver transplantation. Br J Anaesth. 2016;116(4):507-512.

30. Rodríguez - Castro KI, Antonello A, Ferrarese A. Spontaneous bleeding or thrombosis in cirrhosis: What should be feared the most? World J Hepatol. 2015;7(14):1818-1827.

31. Chung WJ. Management of portal hypertensive gastropathy and other bleeding. Clin Mol Hepatol. 2014;20(1):1-5.

32. Chait MM. Lower gastrointestinal bleeding in patients with cirrhosis Arch Dig Disord. 2017;1:14-21.

33. Mallet M, Rudler M, Thabut D. Variceal bleeding in cirrhosis patients. Gastroenterol Rep (Oxf). 2017;5(3):185-192.

34. Violl F, Basili S, Ferro D, et al. Association between high values of D dimer and tissue - plasminogen activator activity and first gastrointestinal bleeding in cirrhotic patients. CALC Group. Thromb Haemost. 1996;76(2):177-183.

35. Goulis J, Patch D, Burroughs AK. Bacterial infection in the pathogenesis of variceal bleeding. Lancet. 1999;353(9147):139-142.

36. Shah NL, Intagliata NM, Northup PG, et al. Procoagulant therapeutics in liver disease: a critique and clinical rationale. Nat Rev Gastroentero Hepatol. 2014;11(11):675-682.

37. Garsia -Tsao G, Abraldes JG, Berzigotti A, et al. Portal hipertensive bleeding in cirrhosis: risk stratification, diagnosis, and management: 2016 practice guidance by the American Association for the study of liver diseases. Hepatology. 2017:65:310-335.

38. Cox HT, Poller L, Thomson JM. Gastric fibrinolysis: a possible etiological link with peptid ulcer. Lancet. 1967;1(7503):1300-1302.

39. Isacson S. Fibrinolysis in the upper gastrointestinal tract. Scand J Gastroenterol. 1987;137:19-21

40. Oka K, Tanaka K. Local fibrinolysis of esophagus and stomach as a cause of haemorrhage in liver cirrhosis. Thromb Res. 1979;14(6):837-844.

41. de Franchis R, Baveno VI Faculty. Expanding consensus in portal hypertension: report of the Baveno VI Consensus Workshop: stratifying risk and individualizing care for portal hypertension. $J$ Hepatol. 2015;63(3):743-752.

42. Under the auspices of the Italian Association for the Study of Liver Diseases (AISF) and the Italian Society of Internal Medicine (SIMI). Hemostatic balance in patients with liver cirrhosis: Report of a consensus conference. Dig Liver Dis. 2016;48(5):455-456.

43. Bosch YP, Weerwind PW, Nelemans PJ, et al. An evaluation of factors affecting activated coagulation time. $J$ Cardiothorac Vasc Anesth. 2012;26:563-568

44. Durrani J, Malik F, Ali N, et al. To be or not to be a case of heparin resistance. J Community Hosp Intern Med Perspect. 2018;8:145-148. 